\section{Eyeing the inflammasome}

\section{By Tim Fulmer, Senior Writer}

Two academic teams have independently shown that targeting the inflammasome reduces retinal damage in mouse models of age-related macular degeneration. ${ }^{1,2}$ One study favors inhibiting inflammasome activation to treat dry AMD, whereas the other suggests boosting it to treat wet AMD. The approach for treating dry AMD has been licensed to iVeena Pharmaceuticals Inc., and the academics focused on wet AMD are developing their own gene therapy.

Inflammasomes are a family of cytosolic protein complexes that consist of three subunits: one of several nod-like receptor proteins (NLRPs), the PYD and CARD domain containing (PYCARD; ASC) protein, and caspase-1 (CASP1). In immune cells, activation of the inflammasome by pathogens triggers release of the proinflammatory cytokines IL-1 $\beta$ and IL-18, which then recruit effector cells to the site of tissue injury or infection as part of the innate immune response.

The inflammasome and its downstream effector cytokines have been implicated in inflammation, cancer and metabolic disorders. ${ }^{3,4}$ However, until now there had been no evidence that the complex plays a role in AMD and other eye diseases.

The new papers suggest that the NLR family pyrin domain containing 3 (NLRP3; NALP3) inflammasome, which consists of NLRP3, ASC and CASP1, mediates the retinal toxicity of two molecules linked to AMD progression: drusen proteins and Alu RNA (see Figure 1, "The inflammasome in age-related macular degeneration"). Moreover, both showed that targeting downstream effectors of the inflammasome reduces retinal damage in mouse AMD models.

\section{To serve and protect}

Drusen are deposits of extracellular proteins that form under the retina and are associated with risk for developing AMD. Despite the correlation between AMD and large drusen deposits in the macula, the underlying mechanism of drusen toxicity has remained unclear.

A team of Irish researchers hypothesized that drusen might cause toxicity through activation of the NLRP3 inflammasome because molecular aggregates, crystals and deposits are known to activate the inflammasome in various other diseases. These include cholesterol crystals in atherosclerosis, ${ }^{5}$ islet amyloid peptide aggregates in type 2 diabetes $^{6}$ and uric acid crystals in gout. ${ }^{7}$

Moreover, excess drusen is known to trigger cellular necrosis, a process that also activates the NLRP3 inflammasome. ${ }^{8}$

To test whether drusen activate inflammasome signaling in the eye, the team first isolated drusen from donor eyes of deceased patients with AMD and added them to cultured human peripheral blood mononuclear cells (PBMCs), which are immune cells that enter the retina during AMD and express the NLRP3 inflammasome.

In those PBMCs, even low concentrations of drusen triggered a significant increase in production of the two main inflammasome effector cytokines-IL-1 $\beta$ and IL-18 - compared with no drusen $(p<0.0001)$. In cultured murine PBMCs, compared with wild-type PBMCs, Nlrp3 knockout led to decreased IL- $1 \beta$ production following addition of drusen.

Thus, drusen-induced upregulation of IL- $1 \beta$ and IL-18 in monocytes required activation of the NLRP3 inflammasome.

Next, the researchers looked at the role of inflammasome activation in the development and progression of AMD.

In a mouse model of wet AMD, knockout of Nlrp3 or Il-18 led to significantly greater choroidal neovascularization $(\mathrm{CNV})$ volume than that in wild-type controls $(p<0.05)$, suggesting that NLRP3 inflammasome activation and IL-18 expression both protected against CNV. Intravitreal injection of an anti-IL-18 antibody also significantly worsened CNV in the mice compared with no treatment $(p=0.0368)$.

The researchers hypothesized that IL-18 might protect against wet AMD by downregulating VEGF, which drives excessive blood vessel production in the retina. Indeed, in a murine brain endothelial cell line, recombinant IL-18 significantly lowered Vegf secretion compared with no treatment $(p<0.05)$.

Taken together, the findings suggest inflammasome activation in immune cells plays a protective role in wet AMD, whereby drusen trigger the NLRP3 inflammasome to upregulate expression of IL-18. The cytokine, in turn, reduces levels of proangiogenic VEGF.

The authors wrote that "a balance may exist whereby a certain focal amount of drusen is tolerated because of its ability to induce IL-18, which in turn may act as an anti-angiogenic effector." They concluded that their findings suggest "strategies aimed at producing or delivering IL-18 to the eye may be beneficial in preventing the progression of $\mathrm{CNV}$ in the context of wet AMD." However, "once a critical level of drusen accumulates, its protective role is negated" and disease sets in, they wrote.

Progression to wet AMD occurs in about $10 \%$ of cases of the dry form, co-lead author Sarah Doyle told SciBX.

Results were published in Nature Medicine.

The study was led by Matthew Campbell, a researcher in the Smurfit Institute of Genetics at Trinity College Dublin, Doyle, a researcher in the Trinity Biomedical Sciences Institute, and Peter Humphries, professor of medical molecular genetics at the Smurfit Institute at Trinity College. Joe Hollyfield, professor of ophthalmology at the Cleveland Clinic Lerner College of Medicine of Case Western Reserve University, isolated the drusen used in the experiments. 
Figure 1. The inflammasome in age-related macular degeneration. Two groups have independently found that the inflammasome and IL-18 play a key role in the development and progression of age-related macular degeneration (AMD).

The inflammasome is a cytosolic, multiprotein complex that triggers the innate immune response to microbial toxins as well as to endogenous proteins, lipids and oligonucleotides.

In a paper published in Nature Medicine, Doyle et al. showed that protein aggregates in the macula known as drusen activated the inflammasome in monocytes [a(1) and $\mathbf{b}(\mathbf{1})]$ to trigger the local production of proinflammatory cytokine IL-18 [c(1)]. The result was downregulation of proangiogenic VEGF and reduced eye damage $[\mathbf{d}(\mathbf{1})$ and $\mathbf{e}(\mathbf{1})]$ in a mouse model of wet AMD.

In a paper published in Cell, Tarallo et al. showed that Alu RNA in the macula plus reactive oxygen species (ROS) activated the inflammasome in retinal pigment epithelial (RPE) cells $[\mathbf{a}(\mathbf{2})$ and $\mathbf{b}(2)]$ to trigger production of IL-18 [c(2)]. The result was activation of myeloid differentiation primary response gene 88 (MYD88), increased RPE degeneration and eye damage [d(2) and e(2)] in a mouse model of geographic atrophy, an advanced form of dry AMD.

Doyle et al. are now developing adeno-associate virus (AAV) vector-mediated delivery of pro-IL-18 gene therapy to enhance IL-18 activity in the eye and treat wet AMD, whereas Tarallo et al. are developing MYD88 inhibitors to block IL-18 activity and treat dry AMD.

\section{The dry path}

In the second paper, a group headed by Jayakrishna Ambati, professor of physiology and vice chair of ophthalmology and visual sciences at the University of Kentucky, found that inflammasome activation worsened retinal damage in geographic atrophy, an advanced form of dry AMD.

The Kentucky researchers followed up on their own 2011 Nature paper that showed that deficiency in the microRNA-processing enzyme dicer 1 ribonuclease type III (DICER1) led to accumulation of Alu RNA molecules in the retinal pigment epithelium (RPE). The result was geographic atrophy and retinal degeneration. ${ }^{9}$

Based on those findings, Ambati and colleagues had originally planned to reduce levels of toxic Alu RNA in the retina using one of two approaches. "We reasoned we could either use Alu antisense therapy to directly lower Alu RNA levels or use DICER1 gene therapy to boost DICER1 levels, which would increase processing of $A l u$ RNA and lower Alu RNA levels," said corresponding author Ambati.

"However, we eventually abandoned both approaches. It proved too difficult to titrate the antisense and gene therapies into the back of the eye without causing dysregulation of RNA processing or unacceptable levels of inflammation," Ambati told SciBX.

Thus, Ambati and colleagues looked downstream of Alu RNA. The challenge was determining which protein Alu RNA initially bound to trigger the toxicity cascade.
The team used a series of knockout mice and cell lines to successively eliminate potential RNA-binding targets such as toll-like receptors (TLRs) and RNA-sensing proteins. For each knockout line, the group compared levels of Alu RNA-mediated RPE degeneration with those in wild-type controls, eliminating from further consideration any protein whose knockout did not result in less degeneration than the wild-type comparator.

By process of elimination, the researchers arrived at the NLRP3 inflammasome and its downstream effector myeloid differentiation primary response gene 88 (MYD88).

Myd88 knockout mice showed no Alu RNA-mediated RPE degeneration compared with wild-type controls, nor did mice with knockout of Nlrp3 or Asc. In wild-type mice, intravitreal delivery of a MYD88 peptide inhibitor prevented RPE degeneration.

Finally, to determine whether the toxicity mechanism had any relevance to patients with $\mathrm{AMD}$, the researchers looked at whether human eyes with Alu RNA-associated geographic atrophy (GA) also showed increased inflammasome signaling. Indeed, NLRP3 and IL18 mRNA levels were significantly higher than those in normal eyes $(p<0.05)$.

The findings suggest inflammasome activation in RPE cells plays a destructive role in dry AMD, whereby Alu RNA triggers the NLRP3 inflammasome to increase the activity of IL-18 and MYD88, which in 
turn leads to RPE degeneration and GA.

"It is reasonable to foresee development of MyD88 inhibitors for prevention or treatment of GA," the authors concluded in their paper in Cell.

\section{Wet and dry issue}

Moving forward, the Trinity team will develop an IL-18 therapy to enhance inflammasome activation in wet AMD, whereas the Kentucky team will develop MYD88 inhibitors to block inflammasome activation in dry AMD.

"Our therapeutic strategy is based on the introduction of pro-IL-18 into the retina using adeno-associated viruses (AAVs)," Trinity co-lead author Doyle told SciBX. Using pro-IL-18, an inactive precursor form of IL-18, "is very important from a safety point of view" because it will limit the activity of the proinflammatory cytokine to only when and where it is needed.

"Our AAV approach would be essentially self-regulating," added co-lead author Campbell. "Pro-IL-18 is converted to its active form only in the presence of the processing enzyme caspase-1, which is expressed in the retina when there is a pathological insult such as excessive drusen prior to CNV development."

But Ambati was skeptical about the prospects for any IL-18-based therapy. "Even if IL-18 suppresses VEGF levels and is antiangiogenic, it would probably not be a viable therapeutic strategy in any stage of AMD, given that our Cell paper shows activation of the inflammasome and production of IL-18 is toxic to the retinal pigment epithelium."

Campbell countered that the findings of the Cell paper apply solely "to end-stage dry AMD, not wet AMD, which is what our paper addresses. We are well aware we are dealing with a proinflammatory cytokine here. Also, it must be stressed that exceedingly small amounts of IL18 are sufficient to have a biochemical effect. We know the challenge moving forward will be determining a level of IL-18 expression that is not damaging to the microenvironment of the retina and yet can keep VEGF levels under control."

Ambati suggested the Trinity group "make a vigorous evaluation of inflammasome and IL-18 activation levels in the eyes of wet AMD patients before pursuing therapeutic strategies along those lines."

It would also be useful "to see if supplying exogenous IL-18 can suppress CNV in an animal model of AMD," said Ryo Kubota, president, chairman and CEO of Acucela Inc.

Acucela's ACU-4429, an oral small molecule visual cycle modulator, is in Phase IIa testing to treat dry AMD. The compound "affects a process upstream of drusen formation and prevents its accumulation," said Kubota.

Campbell said, "In the preclinical workup, we are testing both the AAV approach as well as delivering recombinant IL-18 intravitreally. In addition, we are exploring methods of systemic administration of IL-18 or compounds that could induce expression of IL-18 locally in the eye."
At the moment, Campbell and colleagues are analyzing IL-18 and VEGF levels "not only in the eyes of deceased AMD patients, but we are also trying to analyze the cytokines in vitreous and aqueous samples from living patients with varying stages of AMD," he said.

Meanwhile, iVeena Pharmaceuticals, a biotech cofounded by Ambati in 2006, has exclusively licensed the Cell findings from the University of Kentucky. "The company's plan is to develop intraocular delivery of a MYD88 inhibitor to treat geographic atrophy," said Ambati. "We will pursue that goal along three parallel lines: small molecule screens, siRNAbased therapeutics and peptide-based therapeutics."

Bruce Ksander, assistant professor of ophthalmology at Harvard Medical School, said, "It is unclear to what extent dysfunction of Dicer and the accumulation of Alu RNA occurs in patients with geographic atrophy."

Ambati replied that "out of the few dozen eyes we've studied from geographic atrophy patients, all have had elevated Alu RNA levels. So the phenomenon appears uniform" across the patient population.

Ksander and Patricia D'Amore, professor of ophthalmology and pathology at Harvard Medical School, have found that disruption of lysosomes in RPE cells triggers activation of the NLRP3 inflammasome. Those data have been submitted to an undisclosed peer-reviewed journal.

Ksander also is a scientist at the Schepens Eye Research Institute. D'Amore is co-director of research and a senior scientist at the Institute.

The Nature Medicine findings are patented and available for licensing from Trinity College.

Fulmer, T. SciBX 5(20); doi:10.1038/scibx.2012.511

Published online May 17, 2012

\section{REFERENCES}

1. Doyle, S.L. et al. Nat. Med.; published online April 8, 2012; doi:10.1038/nm.2717

Contact: Matthew Campbell, Trinity College Dublin, Dublin, Ireland e-mail: matthew.campbell@tcd.ie

2. Tarallo, V. et al. Cell; published online April 26, 2012; doi:10.1016/j.cell.2012.03.036

Contact: Jayakrishna Ambati, University of Kentucky, Lexington, Ky.

e-mail: jamba2@email.uky.edu

3. Henao-Mejia, J. et al. Nat. Immunol. 13, 321-324 (2012)

4. Zitvogel, L. et al. Nat. Immunol. 13, 343-351 (2012)

5. Duewell, P. et al. Nature 464, 1357-1361 (2010)

6. Masters, S.L. et al. Nat. Immunol. 11, 897-904 (2010)

7. Martinon, F. et al. Nature 440, 237-241 (2006)

8. Iyer, S.S. et al. Proc. Natl. Acad. Sci. USA 106, 20388-20393 (2009)

9. Kaneko, H. et al. Nature 471, 325-330 (2011)

\section{COMPANIES AND INSTITUTIONS MENTIONED}

Acucela Inc., Seattle, Wash.

Cleveland Clinic Lerner College of Medicine of Case Western

Reserve University, Cleveland, Ohio

Harvard Medical School, Boston, Mass.

iVeena Pharmaceuticals Inc., Salt Lake City, Utah

Trinity Biomedical Sciences Institute, Dublin, Ireland

Trinity College Dublin, Dublin, Ireland

University of Kentucky, Lexington, Ky. 